EPJ Web of Conferences 52, 09010 (2013)

DOI: $10.1051 /$ epjconf/20135209010

(C) Owned by the authors, published by EDP Sciences, 2013

\title{
Heavy neutrino decay at SHALON
}

\author{
V.G. Sinitsyna ${ }^{1}$, a, M. Masip ${ }^{2}$, and V.Y. Sinitsyna ${ }^{1}$ \\ ${ }^{1}$ P. N. Lebedev Physical Institute, Russian Academy of Science \\ ${ }^{2}$ CAFPE and Departamento de Fosica Teoretica y del Cosmos Universidad de Granada
}

\begin{abstract}
The SHALON Cherenkov telescope has recorded over $2 \times 10^{6}$ extensive air showers during the past 17 years. The analysis of the signal at different zenith angles has included observations from the sub-horizontal direction $\Theta=97^{\circ}$. This inclination defines an Earth skimming trajectory with $7 \mathrm{~km}$ of air and around $1000 \mathrm{~km}$ of rock in front of the telescope. During a period of 324 hours of observation, after a cut of shower-like events that may be caused by chaotic sky flashes or reflections on the snow of vertical showers, we have detected 5 air showers of $\mathrm{TeV}$ energies. We argue that these events may be caused by the decay of a long-lived penetrating particle entering the atmosphere from the ground and decaying in front of the telescope. We show that this particle can it not be a muon or a tau lepton. As a possible explanation, we discuss two scenarios with an unstable neutrino of mass $m \approx 0.5 \mathrm{GeV}$ and $c \tau \approx 30 \mathrm{~m}$. Remarkably, one of these models has been recently proposed to explain an excess of electron-like neutrino events at MiniBooNE.
\end{abstract}

\section{Introduction}

Cosmic rays have become a very valuable tool in astronomy, as they provide a very different picture of the sky. In particular, during the past decades gamma-ray detectors have discovered a large number of astrophysical sources (quasars, pulsars, blazars) in our Galaxy and beyond. Ground based telescopes are designed to detect the Cherenkov light of the shower produced when a $0.1-100$ $\mathrm{TeV}$ photon enters the atmosphere. The light burst in a photon (or electron) air shower has a profile that can be distinguished from the one from primary protons or atomic nuclei, which are a diffuse background in such observations (see $[1,2]$ for a review).

Cosmic rays may also offer an opportunity to study the properties of elementary particles. The main objective in experiments like IceCube [3] or Auger [4] is to determine a flux of neutrinos or protons as they interact with terrestrial matter. These interactions involve energies not explored so far at particle colliders, so their study should lead us to a better understanding of that physics. In addition, the size of the detector and its distance to the interaction point is much larger there than in colliders, which may leave some room for unexpected effects caused by long-lived particles. It could well be that in the near future cosmic rays play in particle physics a complementary role similar to the one played nowadays by cosmology (in aspects like dark matter, neutrino masses, etc.)

In this paper we describe what we think may be one of such effects. It occurs studying the response of the SHALON telescope [5] to air showers from different

\footnotetext{
ae-mail: sinits@sci.lebedev.ru
}

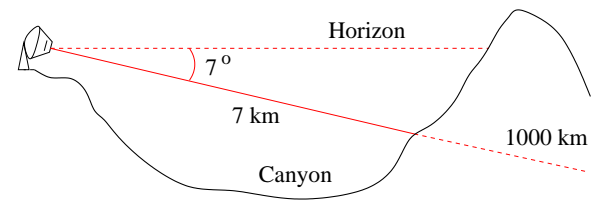

Figure 1. Configuration at $\theta=97^{\circ}$.

zenith angles, in a sub-horizontal configuration where the signal from cosmic rays should vanish.

\section{The SHALON mirror telescope}

SHALON is a gamma-ray telescope [6, 7]. It has been operating since $1992[5,7,8]$. During this period it has detected gamma-ray signals from well known and also from new sources of different type: Crab Nebula, Tycho's SNR, Geminga, Mkn 421, Mkn 501, NGC 1275, SN2006 gy, $3 \mathrm{c} 454.3$ and $1739+522$ [8].

SHALON is located at 3338 meters a.s.l. in the TienShan mountain station. It has a mirror area of $11.2 \mathrm{~m}^{2}$ and a large field of view above $8^{\circ}$, with an image matrix of $144 \mathrm{PMT}$ and $<0.1^{\circ}$ angular resolution. The recording of Cherenkov light is performed in $50 \mathrm{nsec}$ intervals, which is enough to acquire complete information about the air shower while preventing additional light-striking. The telescope is calibrated according to the observations of EAS of cosmic ray at $0^{\circ}$ - zenith angle. The cosmic ray shower image detected in the SHALON telescope, generally is elliptic spot in the light receiver matrix, written in the ADC counts (CODE) (the numbers at fig. 2 right). 
Table 1. The atmosphere depth at different zenith angles.

\begin{tabular}{ccc}
\hline $\begin{array}{c}\text { Zenith } \\
\text { angle, } \Theta^{\circ}\end{array}$ & $\begin{array}{c}\text { Atmosphere } \\
\text { depth, } \mathrm{g} / \mathrm{cm}^{2}\end{array}$ & $\begin{array}{c}\text { Number of Cherenkov } \\
\text { burst per hour }\end{array}$ \\
\hline $0^{\circ}$ & 670 & $1100 \pm 210$ \\
$72^{\circ}$ & 2250 & $7 \pm 1.14$ \\
$76^{\circ}$ & 3000 & $1.8 \pm 0.5$ \\
$84^{\circ}$ & 5950 & $0.5 \pm 0.01$ \\
\hline
\end{tabular}

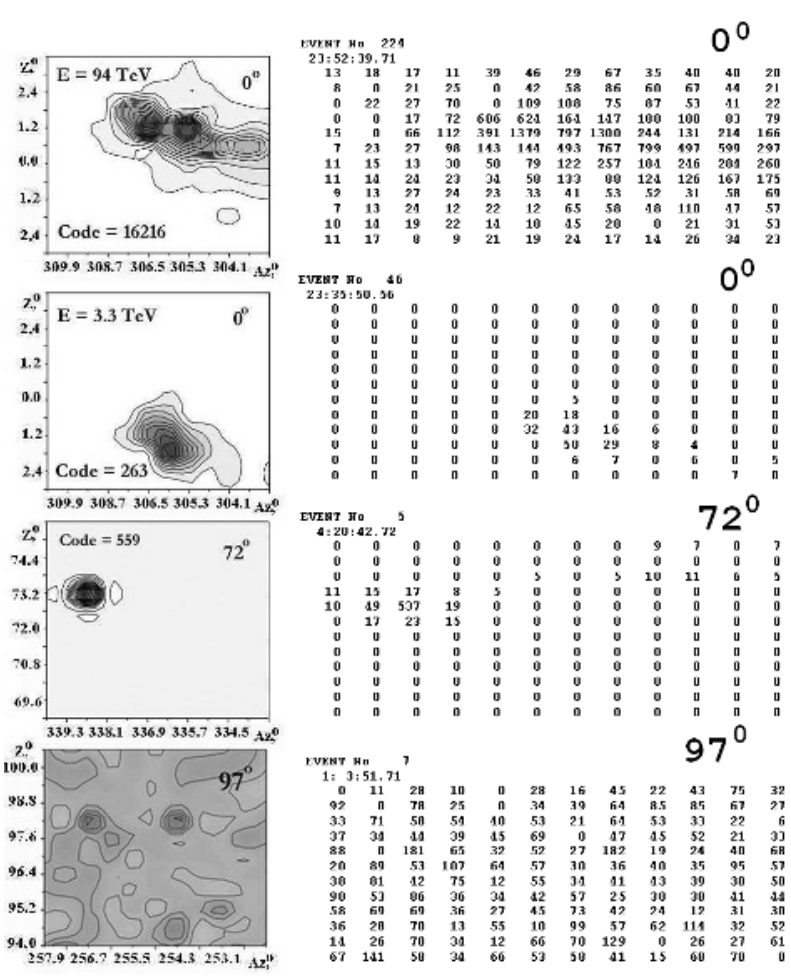

Figure 2. The examples of showers recorded in PMT matrix at different zenith angles. The amplitude of gray - scale shower images is proportional to the ADC count. The number, named CODE shows the range of detected signals in the ADC counts, which are proportional to shower energy.

Observations at large zenith angles have been aimed on study of spectra of the air showers induced by cosmic rays crossing through different atmosphere thickness and events accompanying the passing of EAS and cosmic ray particles near horizon. The observation at large zenith angles $72^{\circ}, 76^{\circ}, 84^{\circ}$ showed that the efficiency of Cherenkov light detection drops essentially as a zenith angle increases, perhaps because of dissipation and absorption in the atmosphere. So, the comparison of observation results shown that at the zenith angle $84^{\circ}$ the number of observed showers is $\sim 25$ times less than expected by estimation with neglecting by absorption and dissipation of Cherenkov photons in the atmosphere (table 1, fig. 2).

\section{Cherenkov bursts below the horizon}

The study of extensive air showers at large zenith angles included observations at the sub-horizontal direction $\theta=97^{\circ}[5,8]$. The configuration of the telescope is depicted in Fig. 1. SHALON Cherenkov mirror telescope is located at $3338 \mathrm{~m}$ a.s.l. The mountain range lies in the east direction and is more than $4300 \mathrm{~m}$ a.s.l. The mountain range is about $20 \mathrm{~km}$ long. The mountain slope has a structure which is irregular on the scales less than typical shower size; it is covered with the forest. The thickness of matter in the telescopic field of view is from 2000 to $800 \mathrm{kms}$; viewed mountain slope area is $>7 \times 10^{5} \mathrm{~m}^{2}$. For telescope located about $7 \mathrm{kms}$ away from the mountain slope horizontally, the shadow of mountain is about $7^{\circ}$ in elevation. In actual conditions the mirror telescope placement the distance till the opposite slope of the gorge is $\sim 7 \mathrm{~km}$ or $\sim 16.5$ radiation units of length, that is quite enough for the development of an electromagnetic cascade till the structure characteristic for the rarefied atmosphere. Observations at $97^{\circ}$ zenith angle have been done in cloudless nights in absence of artificial lights and dry air. During 324 hours of observation at $97^{\circ}$ zenith angle 323 short-range bursts were recorded. In accordance with the existing ideas and estimations $[5,10,11]$ an appearance of electron-photon cascade from upward direction in current SHALON experiment conditions, which are above described, can be connected with passing of weakly interacting particles through rock and earth matter. The identity of upward neutrino initiated showers to cosmic ray ones in frames of concerning experiment has been performed in accordance to the following parameters used in the gamma-ray astronomy. The parameters used to characterize the shower image are image maximum position $\left(x_{\max }, y_{\max }\right)$; length, width; the relation of two previous described Hillas parameters: Length/Width; two parameters sensible to the shower shape: Int $0, \operatorname{Int} 1$; The $\operatorname{Int} 0$ is the ratio of Cherenkov light intensity in pixel with maximum pulse amplitude to the light intensity in the eight surrounding [8]; The Int 1 is the ratio of Cherenkov light intensity in pixel with maximum pulse amplitude to the light intensity in the in all the pixels except for the nine in the center of the matrix. In addition, the selection criteria we are using (Int0, Int 1 , Length/Width) are of the relative nature to describe 2-D shower structure, which is also different in current experiment conditions from vertical one by less than $10 \%$. The parameter proportional to the energy of the shower is Code.

The SHALON databank (since 1992) contains a millions of verified showers from vertical cosmic ray observations with their parameters, so the selection of the showers with a set of parameters of any sample can be performed. Reconstruction of shower coming direction using the analysis of shower shape and position of shower maximum (in case of non-gamma shower) is performed with accuracy $<0.5^{\circ}$ which is enough to judge on whether it upward shower or near horizontal. Horizontal and down going shower is out of field of view because of narrow-beaming of Cherenkov telescope relative to ice or water neutrino telescopes.

During 324 hours of observations 5 events were detected (figs. 3 left) which have expected angular characteristics of a light burst of an electron-photon cascade developing within a telescope observation angle. These showers have energy in the range of about $6-17.5 \mathrm{TeV}$. These 5 events have form characteristics and parameters similar 

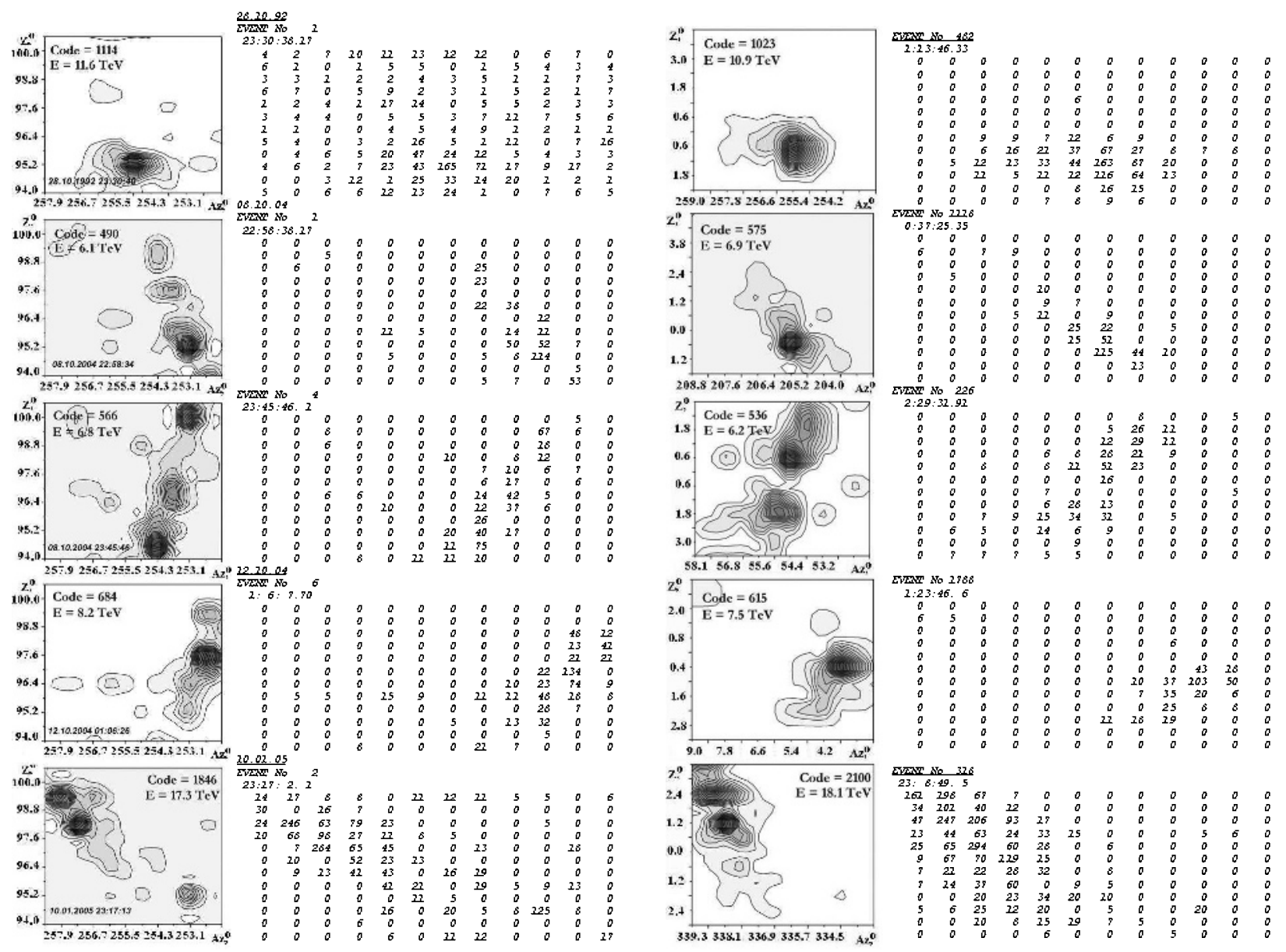

Figure 3. left - Cherenkov Radiation of Extensive Air Showers Observed at $97^{\circ}$ Zenith Angles by SHALON; right - Cherenkov Radiation of Extensive Air Showers Observed at $0^{\circ}$ Zenith Angles by SHALON.

(within $10 \%$ error) to those observed at $0^{\circ}$ zenith angle (figs. 3 right). These cascades look like the usual extensive air showers generated in atmosphere with narrow light shape. The background for this events can be some reflections of cosmic ray EAS in the mountain slope. First of all it could be a reflection of showers initiated by particles born in interaction of very high energy cosmic rays and rock matter nucleons. The energy of detected showers is more than $6 \mathrm{TeV}$. There is no albedo particles of such high energies. One more source of particles with high transverse energy is jet production. The reflection from snow which can mimed the EAS shape is excluded due to the irregular and woody structure of opposite slope (in addition: there is no snow there till the start of November). The probability of hadronic jet production with energy of observed showers is ten orders of magnitude less than one for detection of shower generated by secondary particles of UHE neutrino interaction.

All other 318 events of detection of short-range light bursts in the atmosphere have not a narrow angle light direction and are chaotically distributed along the whole matrix or its part of a light-receiver (see figs. 2 for $97^{\circ}$, no other type of shower image were found among the 318 mentioned pictures). These events may be interpreted as a reflection of a Cherenkov burst from a snow mountain slope or as an ionization luminescence of the atmosphere while an extensive air showers transition within a telescope observation angle.

\section{Earth-skimming neutrino interactions}

The flux of sub-horizontal events is around $6 \times 10^{-6}$ times the flux of $\mathrm{TeV}$ cosmic rays reaching the atmosphere. Such a large flux seems to eliminate the possibility that these events are due to neutrino interactions in the air or within the last $\approx 20 \mathrm{~cm}$ of rock. The interaction length of a 10 $\mathrm{TeV}$ neutrino is $\approx 10^{5} \mathrm{~km}[12]$. This implies that only one out of $10^{9}$ of them will interact to produce such an event. The expected neutrino flux from pion and kaon decays at $10 \mathrm{TeV}$ is a per cent fraction of the primary proton flux, whereas the flux from the prompt decay of charmed hadrons, although uncertain, should be still smaller at these energies [13]. Therefore, the expected number of events from atmospheric neutrino interactions is $10^{5}$ times smaller than the one observed. On the other hand, a flux of primary (non-atmospheric) neutrinos large enough would be inconsistent with observations at neutrino telescopes.

Another possibility that can be readily excluded is the decay in the air of a muon or a tau lepton produced inside the rock. A $10 \mathrm{TeV}$ muon could emerge if it is produced $\approx$ $1 \mathrm{~km}$ inside the rock [12] (one out of $10^{5}$ incident neutrinos will produce a muon there). However, the muon decay 
length at $\mathrm{TeV}$ energies is around $10^{4} \mathrm{~km}$, so the probability that it decays in the air in front of the telescope is again too small. The neutrino fluxes required to explain the events from $\mu$ decays or from $v$ interactions are then similar (and excluded). The probability for tau lepton production in the rock and decay in the air is not higher. The tau becomes long-lived at $\approx 10^{8} \mathrm{GeV}$. At $10 \mathrm{TeV}$ it should be produced within the last meter of rock $(c \tau \gamma \approx 0.5 \mathrm{~m})$, which reduces very much the number of events.

\section{Heavy neutrino decay}

Therefore, we have to explore possible explanations based on new physics. The ideal candidate should be a long-lived massive particle, neutral, frequently produced in air showers, and very penetrating: able to cross $1000 \mathrm{~km}$ of rock and decay within the $7 \mathrm{~km}$ of air in front of the telescope. If this particle has (possibly suppressed) couplings to the $W$ and/or $Z$ bosons, its mass $m_{h}$ should be larger than $m_{\mu}$ (to decay in the last $7 \mathrm{~km}$ of air) and smaller than $m_{\tau}$ (to cross $1000 \mathrm{~km}$ of rock without decaying). Notice that if its decay length at $10 \mathrm{TeV}$ is $c \tau \gamma \approx 1000 \mathrm{~km}$, at $\mathrm{GeV}$ energies it will tend to decay far from the detectors in colliders.

An obvious possibility is a sterile neutrino. We take two Weyl spinors $n$ and $n^{c}$ and add a Dirac mass term together with a Yukawa coupling to the lepton family $L=\left(v_{l} l\right)$,

$$
-\mathcal{L}_{v}=m_{n} n n^{c}+y_{v} h^{\dagger} L n^{c}+\text { h.c. }
$$

Then the Higgs VEV $v$ induces mixing between $n$ and $v_{l}:$

$$
-\mathcal{L}_{v} \supset m_{n} n n^{c}+m_{E W} v_{l} n^{c}=m_{h} v_{h} n^{c},
$$

where $m_{E W}=y_{v} v / \sqrt{2}, m_{h}=\sqrt{m_{n}^{2}+m_{E W}^{2}}, v_{h}=c_{\alpha} n+s_{\alpha} v_{l}$, $s_{\alpha}=m_{E W} / m_{h}$, and the orthogonal combination $-s_{\alpha} n+c_{\alpha} v_{l}$ remains massless. The mixing implies couplings of $v_{h}$ to the $W$ and $Z$ gauge bosons; the first one will appear suppressed by $U_{l h}=s_{\alpha}$, whereas the flavour-changing (heavy to light) $Z$ coupling will be proportional to $c_{\alpha} s_{\alpha}$.

A first $v_{h}$ model that we would like to discuss has been recently proposed by Gninenko [14] to explain an anomaly at MiniBooNE [15]. He claims that the excess of electronlike events in the interactions of the $\langle E\rangle \approx 800 \mathrm{MeV} v_{\mu}$ beam could be caused by the decay of a heavy neutrino if $m_{h} \approx 0.5 \mathrm{GeV}, c \tau_{h} \leq 30 \mathrm{~m}$, and $\left|U_{\mu h}\right|^{2} \approx 10^{-3}$. This explanation requires a large transition magnetic moment [16], $\mu_{\text {tran }} \approx 10^{-10} \mu_{B}$, which implies a dominant decay mode

$$
v_{h} \rightarrow \gamma v \text {. }
$$

The final photon would convert into a $e^{+} e^{-}$pair with a small opening angle that would be indistinguishable from an electron in MiniBooNE. At the same time, this dominant decay channel could make the required value of $U_{\mu h}$ consistent with bounds $\left|U_{\mu h}\right|^{2} \leq 10^{-5}$ from BEBC [17], CHARM [18] and CHARM2 [19], as these experiments look for decays into final states with charged particles $\left(v_{h} \rightarrow e e v, \mu e v, \mu \pi\right)$.

It is easy to see that such a particle could have an impact on the SHALON events. At $10 \mathrm{TeV}$ its decay length is $\lambda_{h} \approx 600 \mathrm{~km}$. If $v_{h}$ is produced in the atmosphere with that energy, the probability that it crosses $\lambda \approx 1000 \mathrm{~km}$ of rock and decays within the $\Delta \lambda \approx 7 \mathrm{~km}$ of air in front of the telescope is

$$
p=e^{-\lambda / \lambda_{h}}\left(1-e^{-\Delta \lambda / \lambda_{h}}\right) \approx 0.002
$$

This implies that the atmospheric flux of heavy neutrinos should be a 1/1000 fraction of the TeV flux of primary cosmic rays. This large flux seems difficult to achieve because $v_{h}$ is not produced in pion or kaon decays (as $m_{h}>m_{\pi, K}$ ), it appears only in a $\left|U_{\mu h}\right|^{2} \approx 10^{-3}$ fraction of charmed hadron decays into muons.

A slightly more frequent production rate could be expected in a second model, where $v_{h}$ has a sizeable component along the tau neutrino. NOMAD [20] has set limits $\left|U_{\tau h}\right|^{2} \leq 10^{-2}$ from $D_{s} \rightarrow \tau v_{h}$, and then $v_{h} \rightarrow v_{\tau} e e$, but they apply only to neutrinos lighter than $m_{D_{s}}-m_{\tau} \approx 0.19$ $\mathrm{GeV}$. Cosmological and supernova bounds on $U_{\tau h}$ apply to lighter values of $m_{h}$ as well [21]. On the other hand, LEP bounds cover just the range $m_{h}>3 \mathrm{GeV}$ [22] (decays in the detector of lighter neutrinos are too rare). Therefore, a possible candidate could have a $0.2-0.4 \mathrm{GeV}$ mass, $\left|U_{\tau h}\right|^{2} \approx 0.1$, and negligible mixings with the other two families. The dominant decay channels would be into $v_{\tau} \pi^{0}$ and into $v_{\tau} e e, v_{\tau} \mu \mu$. If its decay length at the TeV energies of the sub-horizontal events is around $1000 \mathrm{~km}$, then the probability of decay in the air in front of SHALON is $\approx 0.003$. Its production in air showers would be through tau decay; one can expect $\left|U_{\tau h}\right|^{2} \approx 0.1$ heavy neutrinos from each tau produced in the atmosphere. These tau leptons would mainly come from the prompt decay of charmed $D_{s}$ mesons, and also from mesons containing a bottom quark. The flux required, a per cent of the TeV proton flux, seems still too large. Notice, however, that there are also large uncertainties in the flux and energy of the sub-horizontal events, or in the tau production rate in the atmosphere by cosmic rays [13].

\section{Summary and discussion}

When a cosmic ray enters the atmosphere it produces an extended air shower with thousands of secondary particles. Obviously, if there is any new physics it will be contained in a fraction of these events. Now, if this exotic physics includes a long-lived particle, we think that there is the potential for its discovery in cosmic ray experiments. Generically, to be detectable the particle must survive after the rest of the shower has been absorbed by the atmosphere (e.g., a long-lived gluino in horizontal air showers [23]) or the ground (a stau in neutrino telescopes [24]). In particular, a long-lived neutral particle could propagate to the center of a neutrino telescope and start there a contained shower when it decays. However, this event would look indistinguishable from a standard neutrino interaction.

In this paper we discuss several air showers obtained at SHALON in a configuration (see Fig. 1) where the expected number of events is zero. Around $1000 \mathrm{~km}$ of rock absorb the atmospheric flux of any standard particles but neutrinos. Neutrino interactions in the rock are frequent, 
but they are not observable as they disappear in just half a meter of soil. A few muons could be produced during the last $\mathrm{km}$ and emerge from the rock, but then the probability of muon decay within the $7 \mathrm{~km}$ of air in front of the telescope is too small. The crucial difference with a neutrino telescope is that here the probability of a visible $v$ interaction (in the air or the last centimeters of rock) is negligible.

We argue that these events may correspond to the decay of a neutral particle after it is produced in the atmosphere and has crossed $1000 \mathrm{~km}$ of rock. We have studied a couple of models where this particle is a heavy neutrino, and have concluded that although the required production rate seems higher than the expected one, due to a number of uncertainties on the flux and the energy of the exotic events or on the production of charmed particles in the atmosphere, none of these possibilities should be excluded.

Acknowledgements.- The SHALON Experiment has been supported by RFBR 06-02-17364 and by the Program of the Russian Academy of Sciences "Neutrino Physics" 06, 08, 09. The work of M.M. has been supported by MEC of Spain (FPA2006-05294) and by Junta de Andalucía (FQM-101 and FQM-437).

\section{References}

[1] T. C. Weekes, arXiv:0811.1197 [astro-ph].

[2] H. J. Völk and K. Bernloehr, arXiv:0812.4198 [astro$\mathrm{ph}]$.

[3] A. Achterberg, et al. [IceCube Collaboration],: Astropart. Phys. 26, 155 (2006).

[4] J. Abraham, et al., [Pierre Auger Collaboration],: Nucl. Instrum. Meth. A 523, 50 (2004).

[5] V. G. Sinitsyna [SHALON Experiment],: in Proc. of Toward a Major Atmospheric Cherenkov DetectorII, Calgary, July 17-18 1993, ed. R. C. Lamb, 91, (1993).

[6] S. I. Nikolsky and V. G.Sinitsyna [SHALON Experiment],: VANT, Ser. TFE, 1331, 30 (1987).

[7] V. G. Sinitsyna, Prepared for 6th GeV - TeV Gamma Ray Astrophysics Workshop: Toward a Major Atmo- spheric Cerenkov Telescope, Snowbird, Utah, 13-16 Aug 1999.

[8] V. G. Sinitsyna et al., [SHALON Experiment],: Nucl. Phys. Proc. Suppl. 122, 247 (2003); Nucl. Phys. Proc. Suppl. 175-176, 455, 544 (2008); Nucl. Phys. Proc. Suppl. 196, 251, 433, 437, 442 (2009).

[9] V. Y. Sinitsyna et al., [SHALON Experiment],: in Proc. of the 28th International Cosmic Ray Conference, July 31-August 7, 2003. Trukuba, Japan. Editors: T. Kajita et al., 2369, (2004).

[10] L.K. Resvanis, Nucl.Phys.B (Proc.Suppl.) 151, 279 (2006).

[11] D. Fargion, ApJ 570, 909 (2002).

[12] C. Amsler et al., [Particle Data Group],: Phys. Lett. B 667, 1 (2008).

[13] C. G. S. Costa Astropart. Phys. 16, 193 (2001).

[14] S. N. Gninenko, The MiniBooNE anomaly and heavy neutrino decay, arXiv:0902.3802 [hep-ph].

[15] A. A. Aguilar-Arevalo et al., [The MiniBooNE Collaboration],: Phys. Rev. Lett. 98, 231801 (2007).

[16] R. N. Mohapatra and P. B. Pal: "Massive neutrinos in physics and astrophysics. Second edition," World Sci. Lect. Notes Phys. bf60, 1 (1998).

[17] A. M. Cooper-Sarkar et al. [WA66 Collaboration],: Phys. Lett. B 160, 207 (1985).

[18] F. Bergsma et al. [CHARM Collaboration],: Phys. Lett. B 166, 473 (1986).

[19] P. Vilain et al., [CHARM II Collaboration],: Phys. Lett. B 343, 453 (1995).

[20] P. Astier et al., [NOMAD Collaboration],: Phys. Lett. B 506, 27 (2001). [arXiv:hep-ex/0101041].

[21] A. D. Dolgov, S. H. Hansen, G. Raffelt and D. V. Semikoz, Nucl. Phys. B 590, 562 (2000).

[22] O. Adriani et al., [L3 Collaboration],: Phys. Lett. B 295, 371 (1992).

[23] J. I. Illana, M. Masip and D. Meloni, Phys. Rev. D 75, 055002 (2007).

[24] M. Ahlers, J. I. Illana, M. Masip and D. Meloni JCAP 0708, 008 (2007). 